\title{
Cancer - Could it be Cured? A Spontaneous Regression of Cancer, Cancer Energy Metabolism, Hyperglycemia-Hypoglycemia, Metformin, Warburg and Crabtree Effects and a New Perspective in Cancer Treatment
}

\section{Jozef Oleksyszyn ${ }^{1 *}$, Joanna Wietrzyk ${ }^{2}$ and Mateusz Psurski ${ }^{1}$}

${ }^{1}$ Division of Medicinal Chemistry and Microbiology, Faculty of Chemistry, Wrocław University of Technology, PL-50370 Wrocław, Poland

${ }^{2}$ Division Experimental Oncology, Institute of Immunology and Experimental Therapy, Polish Academy of Sciences, PL-53114 Wrocław, Poland

\begin{abstract}
The spontaneous regression of cancer is still a mysterious phenomenon but an understanding of it, at least partially, could lead to new and better cancer treatment. On the basis of new data concerning cancer energy biochemistry, the authors here present a hypothesis to understand and explain the phenomenon of the spontaneous regression of cancer, together with its implications for new approaches to cancer treatment. Such treatment involves the manipulation of the energy metabolism and/or redox status of cancer cells.
\end{abstract}

Keywords: Cancer energy metabolism; Cancer metabolism; Cancer treatment; Cancer cells; Malignant tumor; Tumor regression

\section{Introduction}

The phenomenon of the spontaneous regression of cancer has been observed for a long time. From the outset, it has given rise to a lot of controversy, but now it is accepted as an indisputable fact $[1,2]$. The definition of spontaneous regression is "the partial or complete disappearance of a malignant tumor in the absence of treatment or in the presence of therapy inadequate to affect a significant influence on the disease" [3].

Recently, it has become clear that the phenomenon of spontaneous regression is much more frequent that has been estimated, mainly because of the development of more efficient diagnostic techniques [4-8]. It seems that cases "in the absence of treatment" are rare and probably connected with epigenetic changes that are the effect of diet. Such types of influence are observed for a caloric restriction diet and/ or a ketogenic diet [9]. Cases "in the presence of therapy inadequate to affect a significant influence on the disease" have been known for a long time and include nonspecific immune system stimulation with bacterial vaccination [4]. The vaccine was discovered by Coley and is named Coley's toxin after him $[4,10]$. It is a mixture of heat-killed Streptococcus pyogenes and Serratia marcescens. However, initially, many different strains of bacteria were used and a good therapeutic effect was observed with the infection of tumor patients with diphtheria, gonorrhea, hepatitis, influenza, malaria, measles, smallpox, syphilis and tuberculosis [4-8]. One interesting observation is evident: efficacy is correlated with the degree of fever induced by the toxins/bacteria.

Patients treated with Coley's toxin that develops a fever between $38-40^{\circ} \mathrm{C}$ respond three times better than patients with a lower body temperature [4]. Clearly, the energy crisis of the whole system (due to the fever) has a beneficial effect on the spontaneous remission of cancer tissue. The mechanism of tumor directed stimulation of the immune system is not clear and many hypotheses have been presented, generally focused on the stimulation of cytokine synthesis. Recently, a Phase I trial was initiated with a new and improved biochemicallydefined vaccine, synthesized according to good manufacturing practice and highly promising results have been presented [3]. In this report we would like to compare these data with known literature data concerning carcinogenesis and tumor growth in the attempt to explain the remaining puzzling facts. This should indicate a new approach leading to better anticancer therapies.

\section{A New Look at Carcinogenesis}

It has become clear that cancer cells suffer from a constant shortage of energy supply. Extensive production of reactive oxygen species (ROS) and the required higher antioxidative shield (mainly high concentrations of glutathione) as well as intense proliferation need a large amount of fuel to satisfy cancer cell requirements. At the same time, dysfunction of the mitochondria observed in most, if not all cancer cells [9] limits the amount of ATP synthesized by oxidative phosphorylation (OXPHOS), and induces a few alternative routes for ATP synthesis. These include glycolysis, glutaminolysis and fermentation of other amino acids. Different types of tumor cells use these alternatives to different degrees (because of differences in tumor cells and their environment), as reported in recent papers [10,11]. Any inhibition in even one of these pathways (eg. by calorie restriction, ketogenic diet or partial inhibition of respiratory chains) will result in cancer cell death due energy crisis (apoptosis) [12].

It is a common view that overproduction of ROS is the main cause of carcinogenesis. During the normal process of mitochondrial oxidative phosphorylation, a leakage of ROS, mainly superoxide anions, takes place and it has been estimated that about $0.1-2.0 \%$ of total oxygen consumption is transferred to superoxide anions. Under normal conditions, an antioxidative shield of cells is able to scavenge these by the action of enzymes such as superoxide dismutases (SOD), catalases,

${ }^{*}$ Corresponding author: Oleksyszyn Jozef, Division of Medicinal Chemistry and Microbiology, Faculty of Chemistry, Wrocław University of Technology, PL50370 Wrocław, Poland, Tel: +48 7132040 27; Fax: +48 7132840 64; E-mail: jozef.oleksyszyn@pwr.wroc.pl

Received December 27, 2013; Accepted February 18, 2014; Published February 24, 2014

Citation: Oleksyszyn J, Wietrzyk J, Psurski M (2014) Cancer - Could it be Cured? A Spontaneous Regression of Cancer, Cancer Energy Metabolism, Hyperglycemia-Hypoglycemia, Metformin, Warburg and Crabtree Effects and a New Perspective in Cancer Treatment. J Cancer Sci Ther 6: 056-061. doi:10.4172/1948-5956.1000249

Copyright: (c) 2014 Oleksyszyn J, et al. This is an open-access article distributed under the terms of the Creative Commons Attribution License, which permits unrestricted use, distribution, and reproduction in any medium, provided the original author and source are credited. 

Hyperglycemia-Hypoglycemia, Metformin, Warburg and Crabtree Effects and a New Perspective in Cancer Treatment. J Cancer Sci Ther 6: 056-061. doi:10.4172/1948-5956.1000249

lactoperoxidases, glutathione peroxides, peroxiredoxin and small molecule antioxidants, such as ascorbic acid (vitamin C), tocopherol (vitamins E), uric acid and glutathione. However, the problem becomes seriously dangerous if the shield is broken or when the antioxidant activity of a system is not sufficient to manage to scavenge all the ROS efficiently. In this way, chronic inflammation, aromatic hydrocarbon or viruses induce overproduction of ROS, thus initiating carcinogenesis. To this list, we would like to add hyperglycemia, a more common and physiologically even more relevant and frequently occurring phenomenon. For example, for an obese patient with diabetes, hyperglycemia is common, and both of these diseases are a risk factor for cancer [13]. Physical activity is associated with a preventative effect on cancer, indicating that burning excess energy is here an important factor [14]. Similarly, research on calorie restriction provides evidence that it has a carcinogenesis suppressing activity [15].

Fuel energy overload of the cells has deleterious effects, especially on the mitochondria. Without the synthesis of ATP, the mitochondrial membrane potential becomes extremely negative and the only way to avoid that is the reduction of oxygen to produce oxygen superoxide anions $[16,17]$. The list below illustrates the events which could follow an initial rise in ROS production by the mitochondria:

1. Hyperglycemia

2. Increase in ROS synthesis (also by viruses and carcinogens, e.g. aromatic hydrocarbons, heavy metal cations, etc)

3. Inhibition of Krebs cycle by ROS induced inhibition of aconitase

4. Activation of NF-kappaB

5. Induction of the expression uncoupling protein-2

6. Decrease in mitochondrial membrane potential resulting in suppression of OXPHOS

7. Induction of glycolysis

8. Induction of tumor cell surface NADH oxidases

\section{Hyperglycemia}

The negative associations between hyperglycemia and survival in cancer patients are well documented [18]. Hyperglycemic conditions induce dynamic change in mitochondrial morphology initiating the process of fission. Fission is necessary to increase ROS overproduction [19]. Even a transient high glucose concentration causes persistent epigenetic changes and altered gene expression during subsequent normoglycemia [20]. Finally, a Swedish prospective study documented the association between hyperglycemia and cancer risk. The study included 33,230 women and 31,304 men and the association was independent of obesity [21]. Similarly, hyperinsulinaemia, the sign of hyperglycemia, was found to be a risk factor for lethal clinical prostate cancer [22]. A high glucose concentration also has a deleterious effect on DNA and increase mutagenesis, probably in most human cells [23].

\section{Increase in ROS synthesis}

Cancer tissues are well known to produce increased amounts of ROS [24,25]. Hydrogen peroxide and iron $\mathrm{Fe}^{+2}$ (substrate for the Fenton reaction) are required for the increased mutation of nuclear and mitochondrial DNA. Under such conditions (easy unlimited mutation), cancer cells are able to adopt changes in the gene expression required for survival especially from the energy sources point of view. If the patient already carries the mutation in oncogenes, the process of carcinogenesis is substantially accelerated. Szatrowski [26] has estimated that cancer cells produce hydrogen peroxide in amounts from 0.13 to $0.3 \mathrm{nmol} / 10^{5}$ cells $/ \mathrm{h}$. Similarly, the level of $\mathrm{H}_{2} \mathrm{O}_{2}$ could be measured by estimation of the amount 8-hydroxy-2'deoxyguanosine [27]. Over a longer time interval, the level of hydrogen peroxide could reach a much higher value. The cancer tissue will be sunk in the oxidative environment full of the ROS that accelerated the mutation in the cell DNA.

\section{Inhibition of Krebs cycle by ROS induced inhibition of aconitase}

Mitochondrial aconitase is one of the enzymes from Krebs cycle which contain a $[4 \mathrm{Fe}-4 \mathrm{~S}]^{2+}$ cluster in its active site that catalyse dehydratation-rehydratation of citrate to isocitrate. The enzyme is oxidased by superoxide with the rate constants $\mathrm{k}=10^{7} \mathrm{M}^{-1} \mathrm{~s}^{-1}$ with the formation of hydrogen peroxide and iron II [28]. Both products in the Fenton reaction could form a hydroxyl radical $(\mathrm{OH})$ and accelerate the mutation of mitochondrial DNA (mDNA) $[29,30]$. It needs to be established if that really happens in vivo. However, any decrease in the antioxidative shield of mitochondrium makes that process more likely to occur. Inactivated aconitase could be regenerated by the intracellular reducing agent and thus the Krebs cycle could be only temporally inhibited. Inhibition of aconitase will lead to an accumulation of substrates of the Krebs cycle, such as fumarate, citrate, oxoacetate and succinate causing an inhibition of HIF-alpha (hypoxia inducible factor) prolyl hydroxylase leading to HIF-alpha stabilization under normoxia and which promotes glycolysis $[31,32]$.

\section{Activation of NF-kappaB}

Nuclear factor NF-kappaB is redox sensitive, activated by the ROS transcription factor present in the active form in almost all cancer cells [33]. NF-kappaB plays a pivotal role in the control of a large number of processes, such as immune and inflammatory responses, developmental processes, cellular growth and apoptosis. In addition, it is also responsible for the activation of HIF-alpha genes under normoxia $[34,35]$.

\section{Induction of expression uncoupling protein-2}

As part of the defense response against elevated ROS, the cells induce expression of uncoupling protein-2. There is a link between uncoupling and the carcinogenic process, as has recently been reviewed [36]. The first member of the uncoupling protein family (UCP1) was discovered in brown adipose tissue [37], where it is responsible for uncoupling respiration from ATP synthesis and inducing heat generation. Today, four more UCP homologs have been identified in humans. UCP2 is the most ubiquitous and is frequently cancer-associated [36]. The loss of UCP2 or UCP3 in knockouts yielded an increase in ROS production [38]. However, overexpression increases lifespan in mice mediated by the decreased ROS production and oxidative stress [39]. In general, the part of energy synthesized by the cancer cells came from oxidative phosphorylation OXPHOS. This could be inhibited by the UCP protein and, instead of ATP, heat will be generated. Tumor tissues are known to have higher temperatures than normal tissue [40] and very likely UCP-2 is responsible for this. This topic is still under intense investigation [41]. Any additional uncoupling of mitochondrial membrane potential should put cancer cells in serious energy crisis and induce apoptosis or authopagy [42]. The role of uncoupling proteins in cancer cell mitochondria is still under investigation. a recent paper has suggested that UCP2 expression is necessary for the efficient oxidation of glutamine and may promote the metabolic shift from glucose oxidation to fatty acid oxidation [43] 

Hyperglycemia-Hypoglycemia, Metformin, Warburg and Crabtree Effects and a New Perspective in Cancer Treatment. J Cancer Sci Ther 6: 056-061. doi:10.4172/1948-5956.1000249

\section{Induction of glycolysis}

In response to the hypoxia, cells express several proteins to survive and these include proteins responsible for angiogenesis, apoptosis, $\mathrm{pH}$ regulation, proteolysis, glucose metabolism, cell proliferation/survival and erythropoiesis. Expression of these proteins is under the control of Hypoxia Inducible Factor-alpha (HIF-alpha) and genes are called Hypoxia Response Elements (HRE targeted genes). However, under normoxia all these genes can also be activated, resulting in an induction of glycolysis by the activation of NF-kappaB [33-35] or a high glucose concentration (Carbtree Effect) $[16,17]$.

\section{Induction of tumor cell surface NADH oxidases (NOX)}

All cancer cells express surface-bound NADH oxidase. The protein is present in patient sera due to shedding from cancer cell surfaces [44]. NADH oxidase associated with the neoplastic cells is immunologically and biologically different from NADH oxides associated with normal phagocytic cells; therefore, it has been patented as a general marker for cancer diseases [45]. The enzymes transferred electrons from $\mathrm{NADH}$ to oxygen to produce the superoxide anion. Superoxide anions enzymatically or spontaneously undergo transformation to hydrogen peroxide. It seems that the enzyme is a source of $\mathrm{H}_{2} \mathrm{O}_{2}$ in cancer tissue, as reported earlier $[26,27]$. This is caused by the extensive glycolysis that produces a large amount of NADH and that needs to be oxidized to keep glycolysis running (the Warburg effect). Traditionally, in normal cells, the oxidation of excess of NADH (usually, the NAD+/NADH ratio is around 700) is effected by the lactate dehydrogenase $[46,47]$. The additional part, of cytosolic $\mathrm{NADH}$ is oxidized inside mitochondria where it is transported by the use of redox shuttles including the alphaglycerol-3-phosphate shuttle and the maleate/aspartate NADH redox shuttle. When the mitochondria are dysfunctional or uncoupled [47], this part of the NADH is not oxidized and leads to a decrease in the $\mathrm{NAD}^{+} / \mathrm{NADH}$ ratio; therefore, the NOX protein is expressed and the remaining $\mathrm{NADH}$ is oxidized (the Warburg effect). It seems this last step is an irreversible step for the carcinogenesis. The $\mathrm{NAD}^{+} / \mathrm{NADH}$ ratio controls the activity of sirtuins, $\mathrm{NAD}^{+}$dependent deacetylases that control the expression of many proteins (e.g. histones) [48]. NAD ${ }^{+}$ is a cofactor for these enzymes, but $\mathrm{NADH}$ inhibits the binding of $\mathrm{NAD}^{+}$to the enzyme active site, and in such cases the sirtuins are under much stronger control of the $\mathrm{NAD}^{+} / \mathrm{NADH}$ ratio [49]. Sirtuins control several protein acetylation statuses, including the proteins involved in fatty acid metabolism, ketogenesis, antioxidant enzymes and the PGCalpha cofactor that regulates mitochondrial biogenesis and functions [46].

Usually not recognized, but the changes in the redox status of the cell are the main information network in the organism. Schafer and Buettner measured half-cell reduction potential, $\mathrm{E}_{\mathrm{hc}}$ of the GSSG/2GSH (GSH reduced glutathione) couple, which appeared to correlate with the biological status of the cell [50]. The value of $E_{h c}=-240 \mathrm{mV}$ corresponds to proliferation, $\mathrm{E}_{\mathrm{hc}}=-200 \mathrm{mV}$ to differentiation and $\mathrm{E}_{\mathrm{hc}}=-$ $170 \mathrm{mV}$ to apoptosis. It needs to be established which value corresponds to autophagy. Recent papers [51-53] have indicated that regulation between autophagy and apoptosis is extremely complex. The process is considered a "doubled-edged sword" because autophagy could allow the tumor cells to survive bioenergetics stress. It seems that the choice between autophagy and apoptosis is under the control of redox status [51]. Several redox-sensitive proteins such as cysteine protease Atg4 (oxidation of this block autophagy) [52] and high-mobility group box 1 protein (HMGB1) [51] (reduced protein promote autophagy) are here some of the examples. Again, the level of ROS production at this point is a key factor [53], but the value of $E_{h c}$ characteristic for autophagy needs to be measure. This value probably lies between the half redox potential of these two proteins.

To date, our effort in anticancer drug design has focused on an increase in oxidative stress to induce apoptosis or, in other words, brings the $\mathrm{E}_{\mathrm{hc}}$ to $-170 \mathrm{mV}$. Most anticancer drugs are strong inducers of ROS. However, an identical effect, at least theoretically, could be achieved by the limitations of reductive fuel in amounts to decrease the quantity of the antioxidant shield (e.g. reduced glutathione) to increase oxidative stress, for example, by limiting the supply of carbohydrates, which are the main sources of reductive power (coming from NADPH formed in the glucose phosphate pentose pathway).

The first experiments concerning caloric restriction or ketogenic diet seem to support such an alternative approach [9]. Simultaneously, cancer cells have a high energy requirement due their high proliferation rate and very high reductive shield. For example, human ovarian cells lines showed a 15-50 fold increased level of glutathione (GSH) compared with the drug-sensitive cells of origin [54]. The higher level of GSH in cancer cells seems to be a general feature of tumor tissue $[54,55]$. When glucose is the main supplier of the "reductive power" for the cells, its limited supply through the diet should have a deleterious effect on cancer cell energy metabolism and their redox status. In his excellent books, Seyfried [9] has summarized the data concerning the use of a calorie restricted diet, a ketogenic diet or a calorie restricted ketogenic diet (a diet without carbohydrate or very low carbohydrate contents) as anticancer treatment. However, we are still waiting for the conclusive data from clinical trials. A tumor bearing patient could just accidentally implement the calorie restricted or the ketogenic diet and observe a surprising effect - spontaneous regression of the tumor. This needs to be investigated. However, Nikan, who analyzes more than one thousand reports of spontaneous remission of cancer, concludes that the regression processes are accompanied by hypoglycemia and hypoxia [56]. It is obvious that hypoxia should be a consequence of hypoglycemia because the low glucose levels should impair the erythrocyte activity.

However, the practical use of a ketogenic diet is problematic with respect to processes lowering the glucose level. In our experiments in mice with the use of such diet, we were never able to bring the glucose level below $70 \mathrm{mg} / \mathrm{dl}$. This is due to the action of glucagons, the hormones which at low glucose concentrations start to induce liver gluconeogenesis just to prevent hypoglycemia (this is considered to occur when the glucose level is below $60 \mathrm{mg} / \mathrm{dl}$, however it could be lower and it is individual for each person). Moreover, the ketogenic diet itself induces gluconeogenesis [57]. It seems impossible to induce deep hypoglycemia just by the use of a ketogenic diet or even a calorie restricted ketogenic diet. In fact, in addition to glucagons, many endogenous factors are able to induce gluconeogenesis and these include: fat, growth hormones, exogenous nucleotides, somatostatin, noradrenalin, fructose, and epidermal growth factors [58]. Currently, we do not have any idea as to how deep hypoglycemia needs to be to trigger spontaneous remission. This needs to be investigated if it is possible at all. It is worth mentioning that if gluconeogenesis could be incited by so many factors it is easy to imagine that, locally, hyperglycemia could occur quite frequently, leading to carcinogenesis.

For these reasons, we proposed a new cancer treatment involving the use of a ketogenic diet together with safe and the well-investigated anti-diabetic drug metformin [59]. Our first experiments with 
Citation: Oleksyszyn J, Wietrzyk J, Psurski M (2014) Cancer - Could it be Cured? A Spontaneous Regression of Cancer, Cancer Energy Metabolism, Hyperglycemia-Hypoglycemia, Metformin, Warburg and Crabtree Effects and a New Perspective in Cancer Treatment. J Cancer Sci Ther 6: 056-061. doi:10.4172/1948-5956.1000249

Balb/c bearing $4 \mathrm{~T} 1$ (mouse mammary gland tumor) indicated that simultaneous treatment using metformin ( $1 \mathrm{mg} /$ mouse daily i.p.) and a calorically restricted ketogenic diet (administered at a dosage necessary to meet mice caloric expectations) decreases blood glucose levels to $45 \mathrm{mg} / \mathrm{dl}$ (in contrast to $124 \mathrm{mg} / \mathrm{dl}$ and $75 \mathrm{mg} / \mathrm{dl}$ in a control group receiving standard fodder with no restrictions, and a group receiving calorically restricted diet, respectively) which was associated with a $50 \%$ tumor growth inhibition (TGI) in comparison to the control (38\% TGI) in the case of the group treated with the calorically restricted ketogenic diet. Preliminary data suggest that it could be an effective cancer treatment, but it appears that the treatment schedule needs to be improved and studies need to run on a larger scale facilitating different cancer models (including xenografts) [Psurski M., Wietrzyk J., Oleksyszyn J., unpublished results].

However, the recent investigation of the mechanism of action of metformin led to the discovery that a mild and transitory inhibition of mitochondrial respiratory-chain complex 1 did occur. This led indirectly to the activation of AMP-activated protein kinase (AMPK) and a shut-down of gluconeogenesis. However, the degradation of fatty acids in oxidative phosphorylation of mitochondria will also be suppressed, forming an additional element of the energy synthesis crisis in cancer cells [60]. That effect is probably responsible (together with hypoglycemia) for the anticancer effect of the drug. Therefore, metformin seems to be an ideal candidate to combine its activity with the calorie restricted diet, ketogenic diet or even better with the calorie restricted ketogenic diet.

\section{Spontaneous Remission of Cancer Initiated by Immunomodulation via Bacterial Product Vaccinations}

Most cases of spontaneous remission concern the regression associated with immunostimulation by bacterial product vaccinations (Doley's vaccines) [1-9]. Rapid tumor regression following infection sometimes occurs within hours, suggesting that innate immune response (but not adaptive immune response) is a primary mediator [61]. Provocation of the immune response to induce the spontaneous remission of tumor tissue is the object of intense investigation [1$9,61,62]$.

The mechanism of the immune suppression by the tumor tissue is not clear; however, in 2007 Fisher et al. provided a convincing explanation [63]. The activated T-cells use glycolysis to produce energy after activation. To assure the continuation of glycolysis, the lactic acid molecules, the final product of anaerobic glycolysis, have to be exported by the proton-linked monocarboxylate transporters. Lactate anions are cotransported with the protons following a concentration gradient. Because tumor tissue has a substantially increased lactic acid concentration and lower $\mathrm{pH}$, the transporter is inhibited, leading to suppression of the activation of T-cell and immunosuppression in general. For example, it has been found that human-derived dendritic cells, antigen presenting cells, are also inhibited [64]. In such a scenario, the T-cells approaching the tumor tissue reach low glucose and high lactic acid concentrations and a low $\mathrm{pH}$ environment promoting the stabilization of T-cells in a non-active state. Vaccinations with bacterial products lead to deep hypoglycemia [65-69]. The glucose will be depleted, after a while also causing a decrease in tumor tissue lactic acid concentration. The organism will take care of hypoglycemia and glucose concentrations slowly start to increase. Lactic acid concentrations will still be low (because of the recent hypoglycemia) and under such conditions of rising glucose concentrations the T-cell could be activated. Thus, the activation of the immune system could only happen at a certain ratio between the concentration of glucose and tumor driven-lactic acid, after event of hypoglycemia, when an organism is increasing the glucose level. It is possible that we will never be able to achieve such a "therapeutic window" in vivo, because the system is too dynamic. However, we could relatively easily induce the required changes in glucose concentrations approaching the "therapeutic window". For example, the vaccination with Coley's vaccine required vaccination at least once per week over at least six months to achieve a positive effect [3]. According to the above theoretical considerations, such treatment and experimental data should induce "spontaneous regressions". However, we do not need to use bacterial vaccinations that are always considered to be at least controversial. We can reach "therapeutic windows" by manipulation of the glucose status in an organism. For example, six days on the calorie restricted Ketogenic diet with metformin, as suggested before [59], should help us to replenish glycogen and to lower glucose levels to the amount where the amount of lactic acid produced by the tumor tissue should be as low as possible. It is necessary, during bacterial product vaccination, that the fever replenishes glucose/ATP as heat. After one week, the normal diet (normoglycemia) should be introduced to rapidly increase the glucose levels needed for the activation of the T-cells. The procedure needs to be repeated several times until a positive effect is observed. Many reported cases of spontaneous remission, those without vaccination with the bacterial product, could happen accidentally according to this scenario. These hypotheses need to be verified in clinical trials.

\section{Conclusion}

In conclusion, we propose a general method for cancer treatment, involving the use of cancer energy metabolism and the activation of the immune system by the simple modulation of blood glucose concentrations.

\section{Acknowledgements}

This study was supported by a grant from Wrocław University of Technology, S301 34/20313 and NCN 2011/03/B/ST5/01058.

\section{References}

1. Challis GB, Stam HJ (1990) The spontaneous regression of cancer. A review of cases from 1900 to 1987. Acta Oncol 29: 545-550.

2. Chodorowski Z, Anand JS, Wiśniewski M, Madaliński M, Wierzba K, et al (2007) [Spontaneous regression of cancer--review of cases from 1988 to 2006]. Przegl Lek 64: 380-382.

3. Karbach J, Neumann A, Brand K, Wahle C, Siegel E, et al. (2012) Phase clinical trial of mixed bacterial vaccine (Coley's toxins) in patients with NYESO-1 expressing cancers: immunological effects and clinical activity. $\mathrm{Cl}$ Cancer Res 18: 5449-5459.

4. Jessy T (2011) Immunity over inability: The spontaneous regression of cancer. J Nat Sci Biol Med 2: 43-49.

5. Zahl PH, Maehlen J, Welch HG (2008) The natural history of invasive breast cancers detected by screening mammography. Arch Intern Med 168: 2311 2316.

6. Gladwish A, Clarke K, Bezjak A (2010) Spontaneous regression in advanced non-small cell lung cancer. BMJ Case Rep 2010

7. Bir AS, Fora AA, Levea C, Fakih MG (2009) Spontaneous regression of colorectal cancer metastatic to retroperitoneal lymph nodes. Anticancer Res 29: $465-468$

8. Papac RJ (1998) Spontaneous regression of cancer: possible mechanisms. In Vivo 12: 571-578.

9. Seyfried TN (2012) Cancer as Metabolic Diseases. John Wiley \& Sons, Inc. Hobaken, New Jersey, USA. 
Citation: Oleksyszyn J, Wietrzyk J, Psurski M (2014) Cancer - Could it be Cured? A Spontaneous Regression of Cancer, Cancer Energy Metabolism, Hyperglycemia-Hypoglycemia, Metformin, Warburg and Crabtree Effects and a New Perspective in Cancer Treatment. J Cancer Sci Ther 6: 056-061. doi:10.4172/1948-5956.1000249

10. Jose C, Bellance N, Rossignol R (2011) Choosing between glycolysis and oxidative phosphorylation: a tumor's dilemma? Biochim Biophys Acta 1807 552-561.

11. Dang CV (2012) Links between metabolism and cancer. Genes Dev 26: 877890

12. Zhao Y, Butler EB, Tan M (2013) Targeting cellular metabolism to improve cancer therapeutic. Cell Death and Disease 4: e532.

13. Garg SK, Maurer H, Reed K, Selagamsetty R (2013) Diabetes and cancer: two diseases with obesity as a common risk factor. Diabetes Obes Metab.

14. Pan SY, DesMeules M (2009) Energy intake, physical activity, energy balance, and cancer: epidemiologic evidence. Methods Mol Biol 472: 191-215.

15. Hursting SD, Smith SH, Lashinger LM, Harvey AE, Perkin SN (2010) Calories and carcinogenesis: Lessons learn from 30 years of caloric restriction research. Carcinogenesis 31: 82

16. Diaz-Ruiz R, Rigoulet M, Devin A (2011) The Warburg and Crabtree effects: On the origin of cancer cell energy metabolism and of yeast glucose repression. Biochim Biophys Acta 1807: 568-576.

17. Dell' Antone P (2012) Energy metabolism in cancer cells: how to explain the Warburg and Crabtree effects? Med Hypotheses 79: 388-392.

18. Derr RL, Ye X, Islas MU, Desideri S, Saudek CD, et al. (2009) Association between hyperglycemia and survival in patients with newly diagnosed glioblastoma. J Clin Oncol 27: 1082-1086.

19. Yu T, Robotham JL, Yoon $Y$ (2006) Increased production of reactive oxygen species in hyperglycemic conditions requires dynamic change of mitochondrial morphology. Proc Natl Acad Sci U S A 103: 2653-2658.

20. El-Osta A, Brasacchio D, Yao D, Pocai A, Jones PL, et al. (2008) Transient high glucose causes persistent epigenetic changes and altered gene expression during subsequent normoglycemia. J Exp Med 205: 2409-2417.

21. Stattin $P$, Björ O, Ferrari $P$, Lukanova A, Lenner $P$, et al. (2007) Prospective study of hyperglycemia and cancer risk. Diabetes Care 30: 561-567.

22. Hammarsten J, Högstedt B (2005) Hyperinsulinaemia: a prospective risk factor for lethal clinical prostate cancer. Eur J Cancer 41: 2887-2895.

23. Zhang $Y$, Zhou J, Wang T, Cai L (2007) High level glucose increases mutagenesis in human lymphoblastoid cells. Int J Biol Sci 3: 375-379.

24. Waris G, Ahsan H (2006) Reactive oxygen species: role in the development of cancer and various chronic conditions. J Carcinog 5: 14.

25. Nogueira V, Hay N (2013) Molecular pathways: reactive oxygen species homeostasis in cancer cells and implications for cancer therapy. Clin Cancer Res 19: 4309-4314.

26. Szatrowski TP, Nathan CF (1991) Production of large amounts of hydrogen peroxide by human tumor cells. Cancer Res 51: 794-798.

27. Toyokuni S, Okamoto K, Yodoi J, Hiai H (1995) Persistent oxidative stress in cancer. FEBS Lett 358: 1-3.

28. Vasquez-Vivar J, Kalyanaraman B, Kennedy MC (2000) Mitochondrial aconitase is a source of hydroxyl radical. An electron spin resonance investigation. J Biol Chem 275: 14064-14069.

29. Carew JS, Huang P (2002) Mitochondrial defects in cancer. Mol Cancer 1: 9.

30. Wallace DC, Fan W (2010) Energetics, epigenetics, mitochondrial genetics. Mitochondrion 10: 12-31.

31. Koivunen P, Hirsilä M, Remes AM, Hassinen IE, Kivirikko KI, et al. (2007) Inhibition of hypoxia-inducible factor (HIF) hydroxylases by citric acid cycle intermediates: possible links between cell metabolism and stabilization of HIF J Biol Chem 282: 4524-4532.

32. Selak MA, Armour SM, MacKenzie ED, Boulahbel H, Watson DG, et al. (2005) Succinate links TCA cycle dysfunction to oncogenesis by inhibiting HIF-alpha prolyl hydroxylase. Cancer Cell 7: 77-85

33. Gloire G, Legrand-Poels S, Piette J (2006) NF-kappaB activation by reactive oxygen species: fifteen years later. Biochem Pharmacol 72: 1493-1505.

34. Görlach A, Bonello S (2008) The cross-talk between NF-kappaB and HIF-1: further evidence for a significant liaison. Biochem J 412: e17-19.

35. van Uden P, Kenneth NS, Rocha S (2008) Regulation of hypoxia-inducible factor-1alpha by NF-kappaB. Biochem J 412: 477-484.
36. Valle A, Oliver J, Roca P (2010) Role of uncoupling proteins in cancer. Cancers (Basel) 2: 567-591.

37. Lin CS, Klingenberg M (1982) Characteristics of the isolated purine nucleotide binding protein from brown fat mitochondria. Biochemistry 21: 2950-2956.

38. Vidal-Puig AJ, Grujic D, Zhang CY, Hagen T, Boss O, et al. (2000) Energy metabolism in uncoupling protein 3 gene knockout mice. J Biol Chem 275 16258-16266.

39. Andrews ZB, Horvath TL (2009) Uncoupling protein-2 regulates lifespan in mice. Am J Physiol Endocrinol Metab 296: E621-627.

40. Stefanadis C, Chrysochoou C, Markou D, Petraki K, Panagiotakos DB, et al. (2001) Increased temperature of malignant urinary bladder tumors in vivo: the application of a new method based on a catheter technique. J Clin Oncol 19 : 676-681.

41. Azzu V, Jastroch M, Divakaruni AS, Brand MD (2010) The regulation and turnover of mitochondrial uncoupling proteins. Biochim Biophys Acta 1797: 785-791.

42. Yang ZJ, Chee CE, Huang S, Sinicrope FA (2011) The role of autophagy in cancer: therapeutic implications. Mol Cancer Ther 10: 1533-1541.

43. Samudio I, Fiegl M, Andreeff M (2009) Mitochondrial uncoupling and the Warburg effect: molecular basis for the reprogramming of cancer cell metabolism. Cancer Res 69: 2163-2166.

44. More DJ, Reust T (1997) A circulating Form of NADH oxidase Activity Responsive to the Antitumor Sulfonyl Urea N-4(methylphenylsulfonyl)- $\mathrm{N}$ (4-chlorophenyl)urea LY181984 specific to sera cancer patients. J Bioenerg Biomembr 29: 281-289.

45. Morre DJ, Byrn SR, Crane FL, Morre MD (1997) US Patent 5605810-NADH oxidase assay for neoplasia determination.

46. Houtkooper RH, Cantó C, Wanders RJ, Auwerx J (2010) The secret life of $\mathrm{NAD+}$ : an old metabolite controlling new metabolic signaling pathways. Endocr Rev 31: 194-223.

47. Lu W, Hu Y, Chen G, Chen Z, Zhang H, et al. (2012) Novel role of NOX in supporting aerobic glycolysis in cancer cells with mitochondrial dysfunction and as a potential target for cancer therapy. PLoS Biol 10: e1001326.

48. Li X, Kazgan N (2011) Mammalian sirtuins and energy metabolism. Int J Biol Sci 7: 575-587

49. Teodoro JS, Rolo AP, Palmeira CM (2013) The NAD ratio redox paradox: why does too much reductive power cause oxidative stress? Toxicol Mech Methods 23: 297-302.

50. Schafer FQ, Buettner GR (2001) Redox environment of the cell as viewed through the redox state of the glutathione disulfide/glutathione couple. Free Radic Biol Med 30: 1191-1212.

51. Tang D, Kang R, Cheh CW, Livesey KM, Liang X, et al. (2010) HMGB1 release and redox regulates autophagy and apoptosis in cancer cells. Oncogene 29 5299-5310.

52. Filomeni G, Desideri E, Cardaci S, Rotilio G, Ciriolo MR (2010) Under the ROS...thiol network is the principal suspect for autophagy commitment. Autophagy 6: 999-1005.

53. Mathew R, White E (2011) Autophagy, stress, and cancer metabolism: what doesn't kill you makes you stronger. Cold Spring Harb Symp Quant Biol 76: 389-396.

54. Godwin AK, Meister A, O'Dwyer PJ, Huang CS, Hamilton TC, et al. (1992) High resistance to cisplatin in human ovarian cancer cell lines is associated with market increase of glutathione synthesis. Proc Natl Acad Sci U S A 89: 3070-3074.

55. Singh S, Khan AR, Gupta AK (2012) Role of glutathione in cance pathophysiology and therapeutic interventions. J Exp Ther Oncol 9: 303-316.

56. Nikan B (2010) Spontaneous remission of cancer: Steady and aggressive malignant growth faced with hypoxia and hypoglycemia. Med Hypotheses 75: 505-506.

57. Krebs HA, Speaks RN, Hems R (1965) Accelerations of renal gluconeogenesis by ketone bodies and fatty acids. Biochem J 94: 712-720.

58. Oleksyszyn J (2012) Hyperglycemia and Tumor Energy Metabolism. J Pharmacol Biomed Anal 1: e101. 
Citation: Oleksyszyn J, Wietrzyk J, Psurski M (2014) Cancer - Could it be Cured? A Spontaneous Regression of Cancer, Cancer Energy Metabolism, Hyperglycemia-Hypoglycemia, Metformin, Warburg and Crabtree Effects and a New Perspective in Cancer Treatment. J Cancer Sci Ther 6: 056-061. doi:10.4172/1948-5956.1000249

59. Oleksyszyn J (2011) The complete control of glucose level utilizing the composition of ketogenic diet with the gluconeogenesis inhibitor, the antidiabetic drug metformin, as a potential anti-cancer therapy. Med Hypotheses 77: 171-173.

60. Viollet B, Guigas B, Sanz Garcia N, Leclerc J, Foretz M, et al. (2012) Cellular and molecular mechanisms of metformin: an overview. Clin Sci (Lond) 122: 253-270

61. Hoption Cann SA, van Netten JP, van Netten C (2003) Dr William Coley and tumour regression: a place in history or in the future. Postgrad Med J 79: 672680 .

62. Delmastro-Greenwood MM, Piganelli JD (2013) Changing the energy of an immune response. Am J Clin Exp Immunol 2: 30-54.

63. Fischer K, Hoffmann P, Voelkl S, Meidenbauer N, Ammer J, et al. (2007) Inhibitory effect of tumor cell-derived lactic acid on human T cells. Blood 109: 3812-3819.
64. Lardner A (2001) The effects of extracellular $\mathrm{pH}$ on immune function. J Leukoc Biol 69: 522-530.

65. Amir S, Harel M (1985) Systemic hypoglycemia following central injection of endotoxin in mice. Brain Res 339: 382-385.

66. Caton PW, Nayuni NK, Murch O, Corder R (2009) Endotoxin induced hyperlactatemia and hypoglycemia is linked to decreased mitochondrial phosphoenolpyruvate carboxykinase. Life Sci 84: 738-744.

67. Wolfe RR, Elahi D, Spitzer JJ (1977) Glucose kinetics in dogs following a letha dose of endotoxin. Metabolism 26: 847-850.

68. Witek-Janusek L, Filkins JP (1983) Relation of endotoxin structure to hypoglycemic and insulinlike actions. Circ Shock 11: 23-34.

69. Oguri S, Motegi K, Iwakura Y, Endo Y (2002) Primary role of interleukin-1 alpha and interleukin-1 beta in lipopolysaccharide-induced hypoglycemia in mice. Clin Diagn Lab Immunol 9: 1307-1312. 